\section{REVISTA DE GESTAOO SEECRETARIADO}

MANAGEMENT AND ADMINISTRATIVE

PROFESSIONAL REVIEW

ISSN: 2178-9010
Revista GeSec

São Paulo, SP, Brasil

v. 11, n. 1, p. 106-125

jan. / abr. 2020

DOI: http:// dx.doi.org/ 10.7769/ gesec.v11i1.919

\title{
Governança cooperativa: mapeamento do perfil das publicações científicas internacionais (1991-2018)
}

Cooperative governance: mapping the profile of international scientific publications (1991 - 2018)

\author{
Ramon Rodrigues dos Santos ${ }^{1}$ \\ Joséte Florencio dos Santos ${ }^{2}$ \\ Alexsandra da Silva ${ }^{3}$
}

\section{Resumo}

As cooperativas possuírem estrutura organizacional e objetivos diferentes das organizações tradicionais, uma vez que, pelos princípios doutrinários, assumem a dupla função, de associação e de empresa. Logo, boas práticas de governança cooperativa contribuem para assegurar os interesses dos cooperados, mitigando possíveis hostilidades. Neste aspecto, o presente artigo buscou identificar os elementos que descrevem o perfil das publicações científicas sobre Governança Cooperativa nos periódicos indexados nas bases Scopus no período de 1991 a 2018, contemplando os termos Cooperative Governance e Gobernanza Cooperativa. Os principais resultados mostraram que a produção científica internacional sobre a temática apresenta 94 trabalhos publicados, com um destaque para o período mais recente, de 2016 a 2018, que apresenta o maior número de trabalhos indexados. Em maioria, os artigos são classificados na área Social Sciences e Economics, Econometrics and Finance e são predominantemente produzidos por autores cujas instituições são sediadas nos Estados Unidos e Holanda. Em complemento, identificou-se que a rede de autores é isolada, não havendo cooperação entre os clusters e que os trabalhos citados concentram, dentre as suas palavras-chave, os termos "hazard model", "borrower-dominated", "saver-dominated" e "risco moral".

Palavras-chaves: Bibliometria. Produção Científica. Governança. Cooperativas. Scopus.

\begin{abstract}
Cooperatives have an organizational structure and objectives that are different from traditional organizations, since by doctrinal principles they assume the dual function of association and enterprise. Therefore, good practices of cooperative governance contribute to securing the interests of the cooperative, mitigating possible hostilities. In this aspect, this article sought to identify the elements that describe the profile of scientific publications on Cooperative Governance in periodicals indexed in the Scopus bases from 1991 to 2018, including the terms Cooperative Governance and Cooperative Governance. The main results showed that the international scientific production on the theme presents 94 published works, with a

\footnotetext{
${ }^{1}$ Doutorando em Administração na Universidade Federal de Pernambuco (PROPAD/UFPE).

${ }^{2}$ Vice-coordenadora do Programa de Pós-graduação em Administração da Universidade Federal de Pernambuco (PROPAD/UFPE) e Coordenadora do Mestrado Profissional em Administração (MPA/UFPE).

${ }^{3}$ Mestranda em Administração na Universidade Federal de Pernambuco (PROPAD/UFPE).
} 
highlight for the most recent period, from 2016 to 2018, which presents the highest number of indexed works. Most articles are classified in the area of Social Sciences and Economics, Econometrics and Finance and are predominantly produced by authors whose institutions are based in the United States and the Netherlands. In addition, it was identified that the network of authors is isolated, there is no cooperation among clusters and that the cited works concentrate, among their keywords, the terms "hazard model", "borrower-dominated", "saverdominated "and" moral hazard".

Keywords: Bibliometrics. Cooperatives. Governance. Scientific production. Scopus.

\section{Introdução}

A evolução da concorrência no mercado, a demanda do consumidor, a consolidação da indústria e a política trazem desafios para as cooperativas. Um aspecto importante na resposta das cooperativas modernas a esses desenvolvimentos é a separação entre a administração e a sociedade dos membros. Neste contexto, diante de uma crescente expansão, é imprescindível a necessidade de frameworks definidos e implantados de direção, gestão e respectivas fiscalizações, com fins de proporcionar melhores resultados para estas instituições, visando potencializar o seu desempenho (Peng, Liang, Deng, \& Hendrikse, 2019).

Por esta razão, um conjunto de boas práticas de governança corporativa aplicada às cooperativas, denominadas de "governança cooperativa", contribuem para assegurar os interesses dos cooperados, mitigando possíveis hostilidades. As cooperativas estão entre os tipos de organizações que buscam as melhores práticas de governança e, por tais razões, o aumento no interesse sobre essa questão dentro do universo das mesmas é justificado (Jansen, Maehler \& Wegner, 2018).

O fato é explicado em razão de as cooperativas possuírem estrutura organizacional e objetivos diferentes das organizações tradicionais. Além disso, elas também possuem um conjunto único de disposição de direitos de participação e decisão dos membros, de atividades de controle e de propriedade de gestão (Bialoskorski Neto, Barroso \& Rezende, 2012). De forma geral, o que diferencia a governança em cooperativas da tradicional, que é a corporativa, principalmente, é o fato de que, nas sociedades de pessoas, os clientes também são associados - ou seja, proprietários - e, portanto, possuem interesses quase comuns, o que tende a reduzir o oportunismo (Bosch-Sijtsema \& Postma, 2009).

Neste contexto, optou-se a realizar um estudo bibliométrico sobre a produção científica internacional relacionada à Governança Cooperativa, buscando responder o seguinte questionamento: Quais as características da produção científica sobre Governança Cooperativa nos principais periódicos internacionais no período de 1991 a 2018 ? A ideia 
principal deste trabalho é o de aprofundar a área em estudo, permitindo identificar características essenciais para a produção sobre a temática, através da coleta de dados nos principais periódicos internacionais indexados na Scopus, a partir da produção científica por ano, por autores em produção e citações, áreas do conhecimento, publicação por periódicos, países e afiliação. Ademais, atrela-se a necessidade de aprimorar conhecimento sobre as práticas de governança envolvendo empresas do setor cooperativista, embora constem pesquisas abordando esse tema de forma separada.

Para atingir o problema de pesquisa, este artigo está dividido em mais cinco seções, além desta introdução. O próximo tópico trata do referencial teórico, destacando aspectos relacionados à Governança Cooperativa, à bibliometria e estudos relacionados. No terceiro tópico, os procedimentos metodológicos e a estratégia de coleta. Logo após, os resultados e respectivas análises, considerações finais, e, por fim, as referências.

\section{Referencial Teórico}

\subsection{Governança Cooperativa}

As cooperativas são organizações administrativas por pessoas unidas de forma voluntária para atender suas necessidades e aspirações econômicas, sócias e culturais em comum, baseando-se nos valores de democracia, solidariedade, igualdade e equidade. (International Co-operative Alliance [Coop], 2019). De acordo com Hagendorff (2014), a Governança Corporativa está relacionada aos mecanismos que direcionam o processo decisório dentro de uma organização, lidando com as formas através das quais diversos stakeholders exercem controle sobre a gestão e protegem os seus interesses na tentativa de conciliar os conflitos entre as partes envolvidas.

Neste trabalho, a Governança Cooperativa é considerada como a forma como as organizações gerenciam, delegam e controlam as ações dos seus diversos stakeholders buscando alinhá-las aos diversos interesses e atividades dos cooperados, board e diretoria as quais estão atrelados, conforme a Figura 1. Estes mecanismos são utilizados no intuito de preservar os interesses das partes relacionadas, em que o principal tem controle sob as divergências ao gerenciar as atividades do agente e instituir incentivos mais adequados aos respectivos contratos (Shleifer \& Vishny, 1997).

Seitz (2012) destaca que podem surgir custos referentes à governança, que estariam relacionados ao não cumprimento de contratos firmados entre as partes. Desta forma, os 
estudos de governança se preocupam com a identificação, explicação e minimização das formas de falhas contratuais, e ainda considera que as instituições são consideradas formas de mecanismos de governança, assim como firmas e mercados são encarados como formas alternativas de governança.

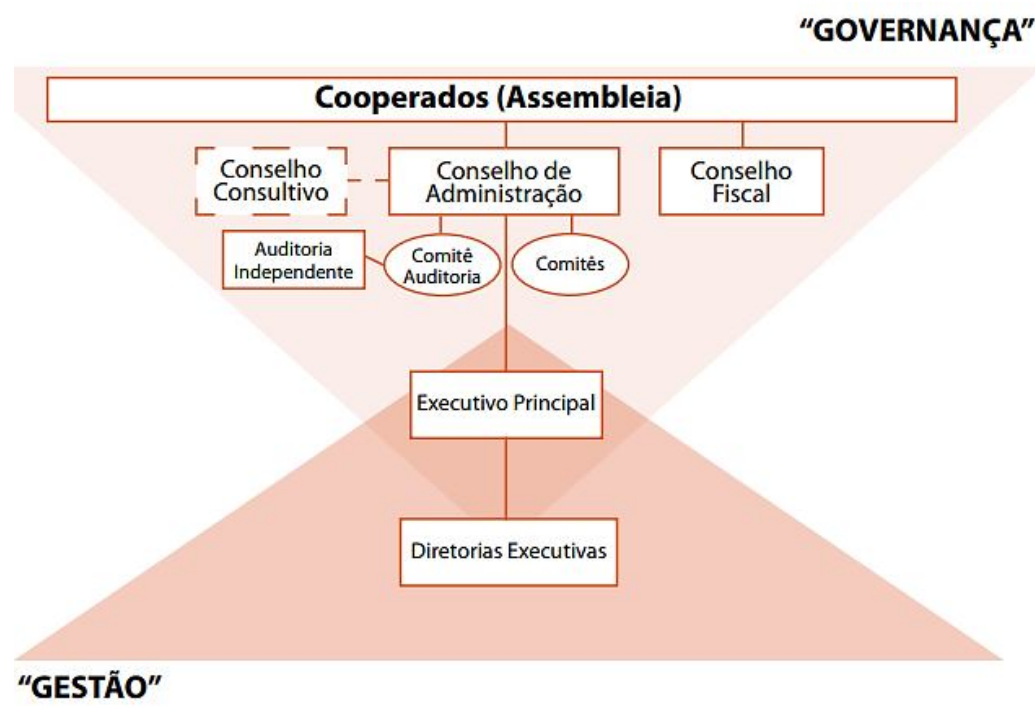

Figura 1. Sistemas de Governança

Fonte: Instituto Brasileiro de Governança Corporativa [IBGC] (2015).

No Brasil, o Guia das Melhores Práticas de Governança para Cooperativas (IBGC, 2015), inspirado nas estruturas existentes e praticadas pelas cooperativas de crédito, saúde e agropecuárias, tem como objetivo, oferecer recomendações que permitam concretizar a eficiência das cooperativas por meio de práticas de governança corporativa, com a finalidade de preservar e otimizar o valor dessas organizações, facilitando seu desenvolvimento e contribuindo para sua longevidade. Já de acordo com a Organização das Cooperativas Brasileiras (Organização das Cooperativas Brasileiras [OCB], 2019), a Governança Cooperativa trata-se de um modelo de direção estratégica, fundamentado nos valores e princípios cooperativistas, que estabelece práticas éticas visando garantir a consecução dos objetivos sociais e assegurar a gestão da cooperativa de modo sustentável em consonância com os interesses dos cooperados.

Essa aproximação entre as propostas de governança e as organizações cooperativas se torna possível devido ao fato de que pelos princípios doutrinários as cooperativas assumem a dupla função, de associação (como reunião de pessoas) e de empresa (como reunião de capital). Da mesma forma, os cooperados ainda assumem um triplo papel: de proprietário, usuário do empreendimento e gestor (Shulze, 1987). 
Neste aspecto, em organizações cooperativas, o processo gerencial tende, por um lado, a não separar necessariamente a propriedade do controle visto que os gestores são os próprios associados. Por outro, considerando que a importância das sobras é pequena em relação à renda anual do cooperado, a sua presença na função gerencial não resulta em menores possibilidades de oportunismo. Pelo contrário, permanecem os custos de agência, que podem afetar o desempenho corporativo (Zylbersztajn, 1994).

\subsection{Estudos Relacionados}

É possível identificar na literatura trabalhos que explorem aspectos relacionados às cooperativas e suas respectivas práticas de governança, com a utilização de outras plataformas e em períodos diferentes. Neste contexto, o presente tópico apresenta os estudos mais recentes.

Duarte, Jesus-Lopes e Santos (2016) buscaram avaliar o perfil das pesquisas que tratem do tema "cooperativas", publicadas entre 2010 e 2014, nos Encontros da Associação Nacional de Pós-Graduação e Pesquisa em Administração (EnANPAD). Os resultados indicam que as áreas de Estratégia nas Organizações, Estudos Organizacionais e Administração Pública são as que mais publicam sobre o tema. Ademais, observou-se que os autores que pesquisam o tema pertencem às universidades brasileiras localizadas nas regiões Sul e Sudeste. Os estudos mostraram a existência de uma maior atenção das pesquisas acadêmicas para estudos empíricos, tais como os estudos de caso e pesquisa de campo. Junto a isso, foi possível verificar a predominância dos temas ligados, nas áreas interdisciplinares que envolvem as logicas da Economia Solidária e do Empreendedorismo, no período pesquisado.

Martins, Lima, Schenatto, \& Bortoluzzi (2017) tiveram como objetivo principal identificar as características das publicações sobre avaliação de desempenho organizacional em cooperativas de crédito nas bases de dados Scielo, Scopus e Web of Science, por intermédio de análise bibliométrica, com acesso em abril de 2017, a partir dos termos "avaliação de desempenho organizacional" e "cooperativas de crédito". Com os resultados foram identificados 15 artigos sobre tema, que, de forma geral, identificaram a falta de consolidação do tema e quanto às metodologias utilizadas demonstrou maior consolidação a metodologia Data Envelopment Analysis (DEA) para avaliar o desempenho de cooperativas de crédito nas bases de dados pesquisadas. 
Silva et al. (2018) buscaram, através de uma pesquisa bibliométrica entre os anos de 1960 e 2017, apresentar a evolução das pesquisas relacionadas a modelos de negócios cooperativos na base Web of Science. A pesquisa aponta para um grande desenvolvimento das pesquisas americanas em relação aos demais países, enquanto que a participação brasileira ainda é muito escassa sobre a temática, o que é uma grande oportunidade para aprofundamentos, tanto na economia, quanto nas pesquisas como um todo. Outro fator relevante é a participação das fontes de fomento chinesas e europeias.

Tomaz et al. (2018) tiveram como finalidade realizar um levantamento bibliográfico, buscando identificar as características bibliométricas de artigos com abordagens no contexto de avaliação de desempenho e eficiência das instituições financeiras, incluindo nestas, as cooperativas de crédito, bancos públicos e privados, evidenciando suas características bibliométricas, cujos dados foram coletados no Portal da Capes e do Google Scholar entre 2009 a 2017. Os resultados evidenciaram as poucas publicações relacionadas ao tema, além de identificar as áreas de Administração, Economia e Contabilidade como as áreas com maior número de publicações. Além disso, identificou-se que a rede de autores é isolada, ou seja, não há cooperação entre os autores do portfólio analisado.

Assim, considerando-se os trabalhos supracitados e as pesquisas realizadas em períodos anteriores, ratifica-se a escassez de estudos que investiguem, de forma conjunta e pontual, a produção científica internacional sobre Governança Cooperativa, considerando a base Scopus para a análise bibliométrica.

\section{Metodologia}

A análise da produção científica contribui para se identificar e visualizar o comportamento das comunidades científicas, instituições, disciplinas, áreas do conhecimento e países, evidenciando sua elite científica, frente de pesquisa, temáticas mais vigorosas, assim como as ligações dentro e entre estas instâncias, entre outros (Almeida \& Grácio, 2019).

Neste aspecto, a bibliometria permite, por meio de um recenseamento, identificar o número de trabalhos sobre um determinado tema, publicados em uma data específica, por um ou mais pesquisadores, em suas respectivas instituições, identificando o que está posto em um determinado campo de conhecimento (Chueke \& Amatucci, 2015). Ademais, pesquisas que empregam a bibliometria tem se tornado frequente entre os pesquisadores, tendo em visto o volume de materiais bibliográficos produzidos e disponibilizados na atualidade. Logo, com 
esse levantamento, é possível facilitar o entendimento para pesquisadores que desejem ingressar em certos campos de pesquisa (Silva et al., 2018).

Quevedo-Silva, Santos, Brandão e Vils (2016) propõem três grupos que agregam diferentes propostas de estudos bibliométricos com a finalidade de classificação e compreensão das diferenças entre estudos e resultados que podem ser esperados. Em um primeiro grupo, as pesquisas descritivas que apresentam em linhas gerais os temas mais estudados em uma área, grupos de pesquisa, periódicos que mais publicam temas correlatos e principais autores e métodos utilizados. Em sequência, os estudos que possuem como foco o domínio metodológico dominante em uma área de pesquisa, preocupando-se em classificar e contabilizar os desenhos de pesquisa mais utilizados no tema e técnicas utilizadas para teste de hipóteses com o objetivo de apontar oportunidades de estudo e destacar a tradição de pesquisa na área.

Por fim, os trabalhos que apresentam as análises descritivas referentes a área de pesquisa conforme objetivo do primeiro grupo de pesquisas descrito. Contudo, avança em relação ao primeiro ao utilizar análises quantitativas dos agrupamentos de teorias, construtos e variáveis operacionalizadas nos estudos, além de ter como objetivo apresentar tendências de pesquisa no tema e proposições teóricas a partir de integração das teorias que suportam o desenvolvimento de cada tema.

Neste contexto, a finalidade do presente trabalho é, ao estar relacionado com a Lei de Zipf e com o primeiro grupo destacado por Quevedo-Silva et al. (2016), apresentar as características da produção científica sobre Governança Cooperativa, cujos registros foram coletados na base de dados Scopus no período compreendido de 1991 a 2018, ou seja, 27 anos. A lógica para o período analisado foi a de considerar desde o primeiro trabalho relacionado ao tema nesta plataforma até o último ano completo. Partindo-se do pressuposto de que trabalhos anteriores não realizaram pesquisas utilizando esta base de dados, este procedimento é prudente para garantir que este trabalho possa ser atualizado sem a necessidade de se fazer novamente a pesquisa completa (Santos, Kalsing \& Hansen, 2014), bastando apenas a realização da pesquisa para um período posterior a 2018.

Quevedo-Silva et al. (2016) destacam que antes de realizar uma bibliometria é importante entender um pouco sobre o tema que será pesquisado para poder definir as palavras-chave. Em um segundo momento, definem-se os filtros de busca. Consoante os autores, as bases de dados mais utilizadas para pesquisas bibliométricas são Web of Science (Thomson Reuters) e Scopus (Elsevier). Ambas as bases já são preparadas para pesquisas bibliométricas com informações sobre número de citações de cada artigo, relevância de 
periódicos, entre outras. A escolha da base Scopus ocorre pelo fato da mesma contemplar artigos apenas em sua versão definitiva, o que implica que essa base não disponibiliza artigos que ainda estão em processo de conclusão, como é o caso de anais de eventos ou artigos cujas revistas já aprovaram, porém com ressalvas (Ferenhof \& Fernandes, 2016).

Este trabalho segue o ritual do processo de filtro dos artigos, apresentado por Lima (2018). Para o presente artigo, a busca foi realizada com os termos Cooperative Governance e Gobernanza Cooperativa, filtrando-se apenas os artigos indexados à base e que apresentassem os respectivos termos apenas em seus títulos. Neste aspecto, para o descritor da base Scopus, a pesquisa foi realizada como “(TITLE (cooperative AND governance) AND TITLE (gobernanza AND cooperative)) AND DOCTYPE (ar) AND PUBYEAR < 2019". O uso dos operadores "AND" (e) e "OR" (ou) possibilita que o pesquisador filtre a sua busca, evitando a separação dos termos, dentro do descritor da base no período analisado.

Com a operacionalização supracitada, os resultados destacados neste trabalho serão baseados no corpus composto por 94 artigos contemplando em seus títulos os termos Cooperative Governance e Gobernanza Cooperativa, publicados nos periódicos indexados na Scopus, no período de 1991 a 2018. Em complemento, realizou-se a análise de autores mais proficientes dentro da temática e redes de coautoria com o suporte do software VOSviewer versão 1.6.10, a partir da importação do arquivo em formato .csv, gerado no site do próprio indexador, no campo Export, opção All (todos).

\section{Resultados}

Este tópico destaca a análise de dados, norteada pelos critérios pré-estabelecidos, relacionados à produção científica por ano, por autores em produção e citações, áreas do conhecimento, publicação por periódicos, países e afiliação. A Figura 2 busca fazer um recorte desses trabalhos por ano de publicação. 


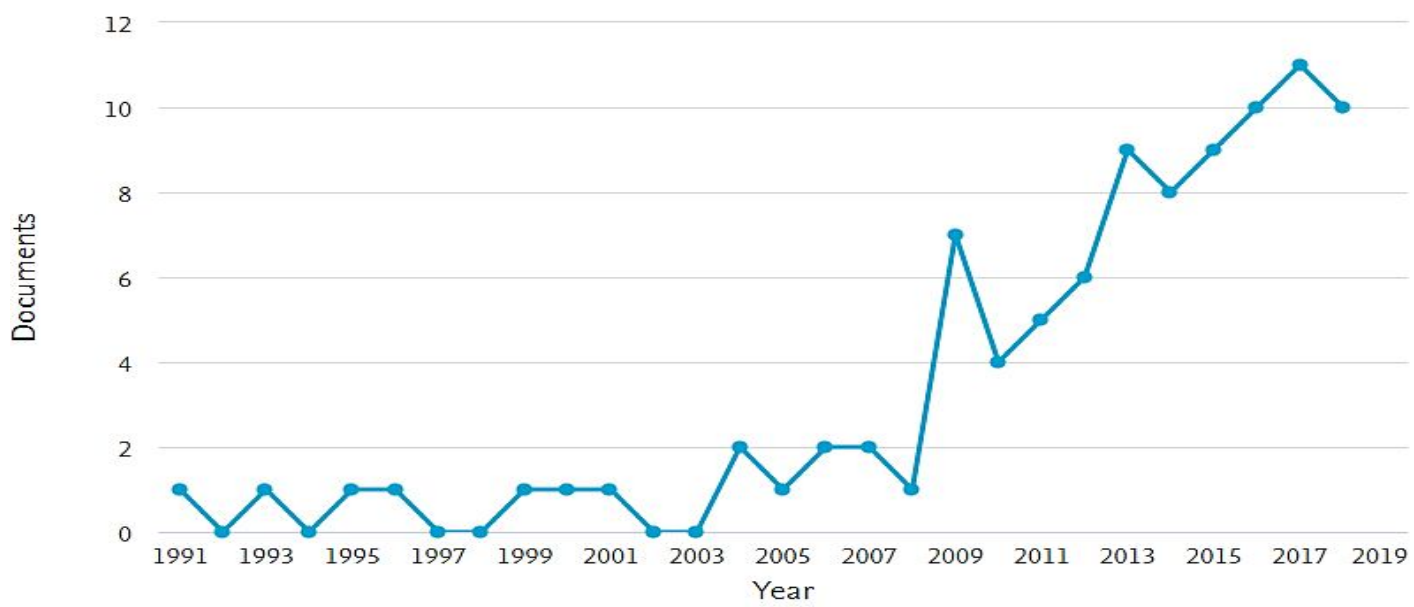

Figura 2. Produção científica sobre governança cooperativa por ano

Fonte: Resultados da Pesquisa, extraídos de Scopus (2019)

Neste recorte, conforme ilustrado na Figura 2, a produção científica sobre governança cooperativa indexadas na base Scopus inicia a partir do ano de 1991, com o trabalho de mesmo título ("Cooperative Governance”), de autoria de Ervin Laszlo, da The Vienna Academy for the Study of the Future, em Viena, na Áustria, cuja publicação foi realizada na World Futures. Ressalta-se que, dos 94 artigos, 87 foram escritos em inglês, 3 em espanhol, 2 em francês e com 1 trabalho/cada, artigos em chinês, alemão e português.

Cabe destacar que o trabalho em português indexado na Scopus é o intitulado Cooperative Governance: The problem of horizon in agricultural cooperative of Rio Grande do Sul (Governança cooperativa: O problema do horizonte em cooperativas agropecuárias do Rio Grande do Sul), publicado na Revista Brasileira de Gestão e Desenvolvimento Regional (G\&DR). O trabalho objetivou analisar o problema em cooperativas agrícolas no Rio Grande do Sul, bem como as estratégias empregadas pelas cooperativas para minimizá-las, optando-se por informações qualitativas e quantitativas em cooperativas agrícolas vinculadas à Organização das Cooperativas do Estado do Rio Grande do Sul (OCERGS) (Pivoto, Waquil, Souza \& Spanhol, 2015).

No Brasil, os registros de estudos sobre cooperativismo de crédito surgiram em meados dos anos 1990, em virtude do desenvolvimento das cooperativas atuantes no contexto atual. Neste aspecto, o cooperativismo de crédito surge como área de estudo no contexto brasileiro de forma recente, tendo como desafio o reconhecimento do modelo de gestão e dos princípios cooperativistas pela comunidade em geral (Freitas \& Freitas, 2014). Com isso, interpreta-se a publicação incipiente de trabalhos brasileiros indexados na Scopus. Ademais, a própria evolução das cooperativas de crédito no Brasil tem o seu marco no ano de 2003, com

Revista Gestão e Secretariado (GeSec), São Paulo, SP, v. 11, n. 1, jan. / abr., 2020, p. 106-125. 
a abertura do crédito cooperativo por meio das Resoluções n. ${ }^{\circ} 3.106$ e n. 3.140 , que ampliaram o alcance dessas instituições aos cidadãos, fomentando a geração de empregos e a distribuição de renda (Souto, 2017).

Em relação ao comportamento das produções internacionais sobre governança cooperativa, a mesma permaneceu incipiente, desde o primeiro ano até o ano de 2008, entre 1 a 2 artigos por ano. Em seguida, a produção cresce exponencialmente, atingindo, para a época, um máximo histórico de 7 artigos, no ano de 2009. A partir deste período, a produção apresenta um decréscimo, para 4 artigos, e logo após, inicia uma evolução, atingindo, em 2017, o máximo histórico entre o período desta pesquisa - em 2018, a produção sobre governança cooperativa indexada na Scopus foi de 10 artigos.

Em sequência, a Figura 3 destaca as principais áreas relacionadas com a produção científica brasileira no período analisado.

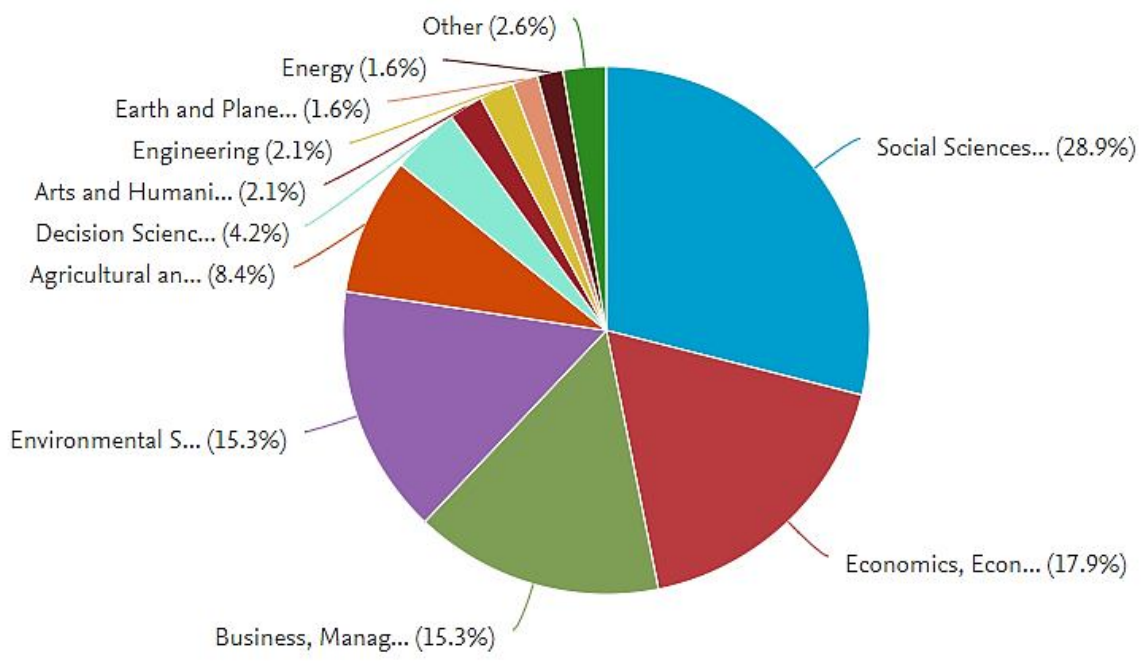

Figura 3. Produção científica sobre governança cooperativa por áreas do conhecimento Fonte: Resultados da Pesquisa, extraídos de Scopus (2019)

A Scopus utiliza a Science Journal Classification Codes (ASJC) e, partindo-se do pressuposto de que os trabalhos indexados na Scopus podem ser classificados em mais de uma área do conhecimento, o somatório dos artigos por área ultrapassa o número de artigos na base, em um total de 190 classificações.

Constata-se pela Tabela 3, que as áreas "Ciências Sociais", "Economia, Econometria e Finanças", "Negócios, Gestão e Contabilidade" e "Ciências Ambientais" concentram aproximados $77,4 \%$ da classificação dos artigos indexados na Scopus. De forma geral, esta 
multidisciplinariedade deve-se à produção internacional sobre governança cooperativa enfatizar este mecanismo em cooperativas agrícolas, rurais e financeiras.

Consoante, o World Cooperative Monitor 2018 (International Cooperative Alliance [Coop], 2018), as cooperativas agrícolas agregam 33\% das instituições mundiais, seguidas pelas que trabalham no segmento financeiro e de seguros $(25 \%)$. Uma cooperativa de crédito é uma instituição financeira de propriedade do associado, controlada democraticamente por seus membros, e operada com o objetivo de maximizar o benefício econômico desses, fornecendo serviços financeiros a taxas competitivas e justas (World Council of Credit Unions [WOCCU], 2019).

A Tabela 1 destaca os periódicos cujos trabalhos que versam sobre governança corporativa foram mais publicados. Para fins de limitação, serão considerados os periódicos que apresentaram dois ou mais trabalhos no período analisado, que representam $27,6 \%$ do corpus desta pesquisa.

Tabela 1

Produção Científica sobre Governança Cooperativa por Periódico e Ano

\begin{tabular}{clcc}
\hline Ordem & Periódico & Quantidade & Ano(s) \\
\hline 1 & Annals of Public and Cooperative Economics & 7 & $2004(2), 2012(2), 2014(2)$, \\
2 & Agribusiness: An International Journal & 4 & $2009(1), 2013(2), 2015(1)$ \\
3 & Land Use Policy & 3 & $2016(1), 2018(2)$ \\
4 & Managerial and Decision Economics & 3 & $2013(2), 2018(1)$ \\
5 & Marine Policy & 3 & $1995(1), 2014(1), 2015(1)$ \\
6 & Ecology and Society & 2 & $2013(1), 2016(1)$ \\
7 & Journal Of Environmental Policy And Planning & 2 & $2011(1), 2013(1)$ \\
8 & REVESCO - Revista de Estudios Cooperativos & 2 & $2011(1), 2017(1)$ \\
\hline
\end{tabular}

Nota. Legenda: “( )": Quantidade de trabalhos publicados no respectivo ano. Fonte: Resultados da Pesquisa (2019)

Conforme destacado, a Annals of Public and Cooperative Economics foi o periódico cujos trabalhos indexados na Scopus foram mais publicados, representando 7 dos 94 artigos, seguido da Agribusiness: An International Journal (4). Fundada em 1908, a "Annals" apresenta trabalhos sobre economia pública e cooperativas com ou sem fins lucrativos, bem como artigos de pesquisa revisando literatura relevante e abordando todas as questões atuais no campo, tanto nos países em desenvolvimento quanto nos desenvolvidos (Annals of Public and Cooperative Economics, 2019). 
Já a Agribusiness: An International Journal enfoca a aplicação da análise econômica à organização e desempenho de empresas e mercados em sistemas industriais de alimentos, incluindo aspectos relacionados a finanças e análise de política pública (Agribusiness, 2019). Diante do cenário, ratifica-se a classificação dos artigos nas áreas destacadas na Figura 3.

A Tabela 2 identifica as cinco principais produções científicas a partir da relação artigo/afiliações, ou seja, as instituições declaradas pelos autores para o respectivo artigo, de forma cumulativa. Ressalta-se que os trabalhos sobre governança cooperativa indexados na Scopus foram escritos por autores de 154 afiliações. Diante disso, as instituições que apresentaram apenas uma afiliação/artigo foram suprimidas.

Tabela 2

Produção Científica sobre Governança Cooperativa por Instituição

\begin{tabular}{|c|l|c|}
\hline Ordem & Periódicos & $\begin{array}{c}\text { Produções/ } \\
\text { Instituição }\end{array}$ \\
\hline 1 & $\begin{array}{l}\text { Wageningen University and Research Centre, Erasmus University Rotterdam, } \\
\text { Rotterdam School of Management }\end{array}$ & 4 \\
\hline 2 & $\begin{array}{l}\text { Zhejiang University, University of Missouri-Columbia, National University of } \\
\text { Singapore }\end{array}$ & 3 \\
\hline 3 & $\begin{array}{l}\text { University of Cape Town, Humboldt-Universität zu Berlin, Universidade Federal de } \\
\text { Mato Grosso do Sul, Open University, Hangzhou Dianzi University, Radboud } \\
\text { University Nijmegen, Universidad de Zaragoza, University of Melbourne, Ludong } \\
\text { University, EURAC Research, NUS - Lee Kuan Yew School of Public Policy }\end{array}$ & 2 \\
\hline
\end{tabular}

Nota. Fonte: Resultados da Pesquisa (2019).

De acordo com a Tabela 2, destaca-se a predominância da Wageningen University and Research Centre (WUR), da Erasmus University Rotterdam e da Rotterdam School of Management, cujas sedes são na Holanda, e, em complemento, os trabalhos afiliados à Zhejiang University, na China, na University of Missouri-Columbia, nos Estados Unidos e da National University of Singapore, em Cingapura, com 3 afiliações.

Junto às demais instituições apresentadas na Tabela 2, que apresentaram 2 afiliações indexadas, as 17 supracitadas representaram 27,9\% do corpus da pesquisa. Em geral, demonstra-se que as pesquisas sobre governança cooperativa são apresentadas de forma pulverizada enquanto afiliações. Não obstante, quando a produção é analisada por países/territórios, identifica-se uma concentração dos trabalhos realizados por autores institucionalizados nos Estados Unidos, Holanda e China, consoante a Figura 4. 


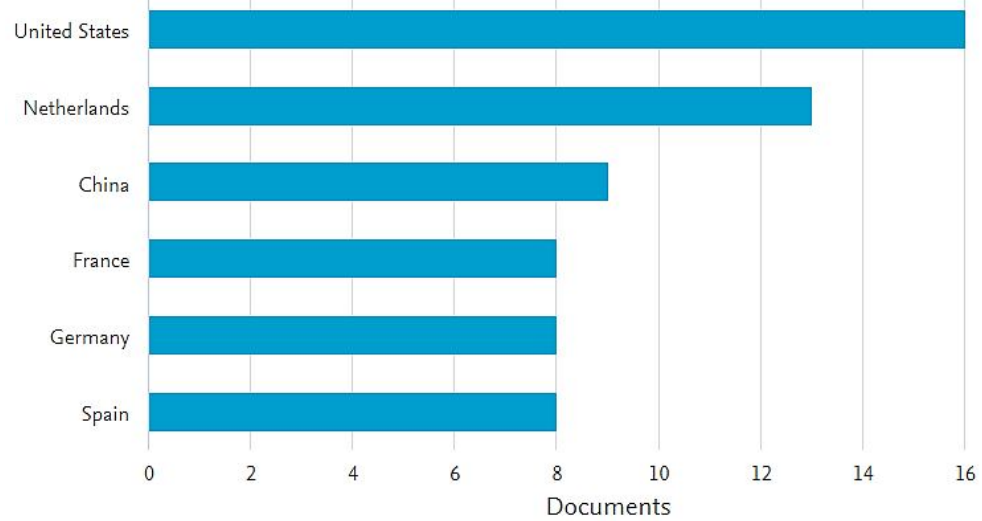

Figura 4. Produção científica sobre governança cooperativa por países/territórios

Fonte: Resultados da Pesquisa, extraídos de Scopus (2019)

Pela análise da Figura 4, identifica-se que os trabalhos realizados pelos seis primeiros países listados representam 40,2\% do total de 154 indexadas na Scopus, destacando que, apenas os artigos cujas instituições são sediadas nos Estados Unidos e Holanda, somadas, representam 18,9\%, ratificando os aspectos apresentados na Tabela 2. Em sequência, a Tabela 3 apresenta a relação dos autores que mais publicaram sobre Governança Cooperativa em periódicos indexados na Scopus durante o período analisado. A tabela ilustra os autores que apresentam dois ou mais registros na base Scopus.

Tabela 3

Principais Autores

\begin{tabular}{clccc}
\hline Ordem & Autores & Instituição, País & Quantidade & Citações \\
\hline 1 & Liang, Qiao & China Academy for Rural Development, China & 3 & 27 \\
2 & Hendrikse, George & Université de Rennes, França & 3 & 25 \\
3 & Ruben, Ruerd & Radboud University Nijmegen, Holanda & 2 & 31 \\
4 & Marcuello, Carmen & University of Zaragoza, Espanha & 2 & 3
\end{tabular}

Nota. Fonte: Resultados da Pesquisa, extraídos do VOSviewer (2019).

De acordo com a Tabela 3, Qiao Liang e George Hendrikse foram os autores que mais produziram sobre governança cooperativa, com 3 trabalhos, seguidos de Ruerd Ruben e Carmen Marcuello, com dois. Cabe ressaltar que, mesmo diante de um número incipiente de trabalhos, o número de citações concentradas nestes quatro países corrobora que a questão do cooperativismo e sua gestão vem ganhando destaque nos últimos anos, tanto em países ocidentais como em países asiáticos, em especial a China, onde existem mais de 689 mil cooperativas agrícolas, com mais de 43 milhões de membros (Jansen, Maehler \& Wegner, 
2018). Em busca de analisar as redes de cooperação entre os autores que compuseram o corpus dessa pesquisa, gerou-se o gráfico ilustrado na Figura 5.

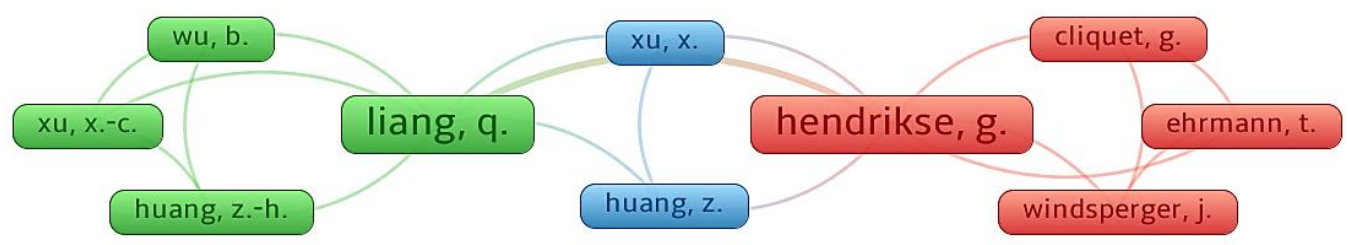

Figura 4. Redes de cooperação nas pesquisas sobre governança cooperativa na scopus

Fonte: Resultados da Pesquisa, extraídos do VOSviewer (2019)

Conforme a Figura 4, a rede de autores é isolada, não havendo cooperação direta entre os clusters e com trabalhos que variam de 2 a 4 autores. $\mathrm{O}$ primeiro cluster é formado por $\mathrm{X}$. Xu e Z. Huang, da China Academy for Rural Development, que estabelecem a relação indireta com os demais clusters. O segundo cluster é composto por B. Xu e Q. Liang, também da China Academy for Rural Development, China. Por fim, o terceiro cluster é composto por G. Cliquet, G. Hendrikse, T. Ehrmann e J. Windsperger, ambos da Université de Rennes, França.

A respeito das publicações mais citadas, é possível verificar tal informação na Tabela 4. Ressalta-se que são apresentados os registros que tiveram mais de 50 citações.

Tabela 4

Publicações mais citadas

\begin{tabular}{clc}
\hline Ordem & Autores/Título/Periódico/Volume/Número/Ano/Páginas & Citações \\
\hline 1 & $\begin{array}{l}\text { Cornforth, C. The Governance of cooperatives and mutual associations: A paradox } \\
\text { perspective. (2004). Annals of Public and Cooperative Economics, 75 (1), pp. 11-32. }\end{array}$ & 147 \\
2 & $\begin{array}{l}\text { Hart, O., Moore, J. The governance of exchanges: Members' cooperatives versus outside } \\
\text { ownership. (1996). Oxford Review of Economic Policy, 12 (4), pp. 53-69. }\end{array}$ & 125 \\
& Bosch-Sijtsema, P. M., Postma, T. J. B. M. Cooperative innovation projects: Capabilities \\
& and governance mechanisms. (2009). Journal of Product Innovation Management, 26 (1), \\
& pp. 58-70. & 69 \\
& $\begin{array}{l}\text { Österberg, P., Nilsson, J. Members' perception of their participation in the governance of } \\
\text { cooperatives: The key to trust and commitment in agricultural cooperatives (2009). }\end{array}$ & 68 \\
& $\begin{array}{l}\text { Agribusiness, 25 (2), pp. 181-197. } \\
\text { Hoekstra, A.Y. The global dimension of water governance: Why the river basin approach }\end{array}$ \\
& $\begin{array}{l}\text { is no longer sufficient and why cooperative action at global level is needed. (2011). } \\
\text { Water (Switzerland), 3 (1), pp. 21-46. }\end{array}$ \\
\end{tabular}

Nota. Fonte: Resultados da Pesquisa (2019).

O trabalho intitulado "The Governance of cooperatives and mutual associations: A paradox perspective", de autoria de Chris Cornforth, com 147 citações registradas, buscou apresentar, de forma seminal, uma nova estrutura teórica para entender a governança de 
organizações cooperativas e mútuas, visto que, há época, era pouco desenvolvida e desconsiderando o framework destas instituições.

$\mathrm{O}$ artigo analisa brevemente algumas das principais perspectivas teóricas sobre governança corporativa e discute como elas podem ser utilmente estendidas para esclarecer a governança das cooperativas e mútuas. Entretanto, individualmente, essas teorias diferentes são unidimensionais, apenas iluminando um aspecto particular do papel do conselho, levando a pedidos por uma nova estrutura conceitual que possa ajudar a integrar os insights dessas diferentes teorias. Neste contexto, nas cooperativas de crédito, a alta dependência da confiança dos associados e a alta exposição ao risco de imagem apontam para a necessidade de práticas que possibilitem a continuidade, objetivando reforçar a confiança e credibilidade dessas instituições (Carvalho et al., 2015).

$\mathrm{O}$ segundo trabalho apresentado na Tabela 4, intitulado "The governance of exchanges: Members' cooperatives versus outside ownership", de Oliver Hart e John Moore destacam que a tomada de decisão coletiva é tipicamente ineficiente, porque em uma votação os pontos de vista do eleitor principal não são necessariamente os mesmos que os da sociedade como um todo. Assim, as duas formas de governança, a propriedade externa e a cooperativa de membros são ineficientes - mas por razões diferentes e de maneiras diferentes. Ao comparar as duas estruturas de propriedade, descobrimos que a propriedade externa tornase relativamente mais eficiente do que a cooperativa de um membro como: (i) a variação entre os membros torna-se mais distorcida; e (ii) o câmbio enfrenta mais concorrência.

De acordo com os autores, para muitas trocas, mas essas mudanças ocorreram nos últimos anos. Entretanto, mesmo que a propriedade externa seja agora a estrutura superior de governança para uma troca, a cooperativa de membros não pode, por sua vontade, votar a favor de fazer a mudança; ou seja, eles podem decidir não vender. Isso ocorre porque os ganhadores podem ser incapazes de compensar os perdedores. Cabe ressaltar que as cooperativas possuem uma estrutura de propriedade pulverizada, divergindo da concentração das empresas tradicionais que atuam no cenário brasileiro, que são caracterizadas como altamente concentrada (Peng et al., 2019)

Com 69 citações, o artigo "Cooperative innovation projects: Capabilities and governance mechanisms", de autoria de Petra Bosch $\square$ Sijtsema e Theo Postma, teve como finalidade o compartilhamento de capacidades em projetos cooperativos de inovação e como essas cooperações são governadas, através de um conjunto de proposições. Em primeiro lugar, uma cooperação destinada a um benefício estratégico mútuo para obter acesso mútuo às bases 
de conhecimento das empresas envolvidas mantém sua própria base de conhecimento e resultando em uma confiança mútua entre as empresas cooperantes.

Em segundo lugar, em uma cooperação voltada para um benefício estratégico mútuo, os parceiros não só obtêm acesso às capacidades tecnológicas do outro, como também desenvolvem e compartilham conhecimento sobre aspectos organizacionais e situações de mercado. Terceiro, em uma cooperação voltada para o benefício estratégico mútuo de acesso mútuo às bases de conhecimento das empresas envolvidas, a não-codificabilidade das capacidades é condicional para criar uma situação de ganho mútuo. E, por último, em quarto, a cooperação voltada para um benefício estratégico mútuo no acesso mútuo às bases de conhecimento das empresas envolvidas baseia-se na competência mútua e na confiança intencional como seu principal mecanismo de governança.

Cheney et al. (2014) alegam que as sociedades de pessoas possuem a função de reestruturar o mercado econômico como um todo, além de promoverem formas e mecanismos alternativos de governança. O fato é explicado em razão de as cooperativas possuírem estrutura organizacional e objetivos diferentes das organizações tradicionais. Com isso, o artigo "Members' perception of their participation in the governance of cooperatives: The key to trust and commitment in agricultural cooperatives" de Peter Österberg e Jerker Nilsson, explora a medida em que a avaliação dos membros sobre o grau de sucesso de suas cooperativas está relacionada a vários atributos dos membros, notadamente a percepção dos membros de sua participação na governança das cooperativas. Três categorias de atributos de membros são identificadas: satisfação com a lucratividade das operações da fazenda, idade do membro e experiência do trabalho na diretoria. O grau de sucesso das cooperativas é medido pelo comprometimento dos membros com as cooperativas e pela confiança dos membros no conselho de administração.

Em complemento, identifica-se que os trabalhos citados concentram, dentre as suas palavras-chave, os termos "hazard model", "borrower-dominated", "saver-dominated" e "risco moral". É importante ressaltar que as estruturas de propriedade e de gestão de risco das cooperativas de crédito e as consequentes relações entre cooperados e cooperativa são peculiares às demais instituições financeiras. No caso das cooperativas de crédito, as relações de conflitos de agência são específicas para esse tipo de organização, em uma perspectiva principal-principal, pois todos os cooperados são proprietários, e, somente com a utilização dos serviços e da participação do cooperado é que a cooperativa se tornará mais forte e capaz de oferecer melhores serviços (Trindade \& Bialoskorski Neto, 2014).

Revista Gestão e Secretariado (GeSec), São Paulo, SP, v. 11, n. 1, jan. / abr., 2020, p. 106-125. 


\section{Considerações Finais}

A Governança Cooperativa busca lidar com questões resultantes de problemas de agência, decorrentes da complexidade e singularidade de sua gestão. Neste aspecto, o presente estudo teve como objetivo analisar a produção científica brasileira indexada na base Scopus no período de 1991 a 2018, considerando o primeiro trabalho relacionado à temática, até o último ano completo. A ideia deste trabalho foi a de apresentar as principais características pertencentes aos trabalhos sobre a temática em termos de ano de publicação, áreas do conhecimento, periódicos cujos artigos foram publicados, instituições, autores mais citados e redes de cooperação entre os autores que compuseram o corpus desta pesquisa.

Por meio deste trabalho, constatou-se que a produção científica indexada na Scopus ainda é incipiente, com 94 trabalhos publicados, cujo ano de 2017 concentrou aproximados $12 \%$ desta produção (11). Quando considerada a produção científica internacional sobre a temática, os artigos cujas instituições são sediadas nos Estados Unidos e Holanda, somadas, representam 18,9\% (29 de 154 indexações). Em maioria, os artigos são classificados na área "Ciências Sociais", "Economia, Econometria e Finanças", "Negócios, Gestão e Contabilidade" e "Ciências Ambientais", visto que, em geral, os trabalhos inseridos tratam sobre o desempenho econômico-financeiro das entidades e a concorrência entre estas no mercado financeiro ou agropecuário.

Em sequência, destacou-se a predominância em produção e citações de instituições chinesas, holandesas e americanas, fato este que já foi corroborado em estudos anteriores sobre a temática. Em complemento, identificou-se que a rede de autores é isolada, não havendo cooperação entre os clusters e com trabalhos que variam de 2 a 4 autores. Por fim, a partir dos trabalhos indexados, mostrou-se que dos 94 trabalhos publicados, poucos possuem acima de 50 citações no período, sendo assim, pulverizados, o que pode ser justificado pelas temáticas específicas.

Os resultados obtidos nesta pesquisa contribuem a outros trabalhos e autores que trabalharam como bibliometria aplicada a temas adjacentes às cooperativas, bem como apresenta o panorama internacional da produção cientifica sobre a temática em um indexador que apresenta trabalhos nacionais e internacionais de alto fator de impacto, como a Scopus.

A principal limitação desta pesquisa e dos resultados apresentados está relacionada ao indexador utilizado, visto que apenas a base Scopus foi aplicada para este artigo, sugerindo-se que, em futuras pesquisas, outros indexadores, como a Web of Science e/ou Scielo sejam utilizados, com a finalidade de promover meta-análises ou possíveis revisões sistemáticas, 
buscando tendências de pesquisa sobre a temática. Além disso, sugerem-se trabalhos que abordem de forma pontual a produção científica sobre cooperativas de crédito em âmbito nacional e internacional, buscando estabelecer, inclusive, revisões sistemáticas ou metaanálises sobre os principais temas ou palavras-chave.

\section{Referências}

Agribusiness: An International Journal. (2019). Overview. Recuperado em 13 de abril, 2019, de https://onlinelibrary.wiley.com/journal/15206297.

Almeida, C., \& Gracio, M. (2019). Produção científica brasileira sobre o indicador "Fator de Impacto": um estudo nas bases SciELO, Scopus e Web of Science. Encontros Bibli: Revista Eletrônica de Biblioteconomia e Ciência da Informação, 24(54), 62-77.

Annals of Public and Cooperative Economics. (2019). Overview. Recuperado em 13 de abril, 2019 , https://onlinelibrary.wiley.com/page/journal/14678292/homepage/productinformation.ht $\mathrm{ml}$.

Baker, H. K., \& Anderson, R. (2010). Corporate governance: a synthesis of theory, research, and practice. The Robert W. Kolb series in finance. New Jersey: Wiley.

Bialoskorski Neto, S.; Barroso, M. F. G.; Rezende, A. J. (2012). Co-operative governance and management control systems: an agency costs theoretical approach. Brazilian Business Review, 9 (2), 68-87.

Bosch $\square$ Sijtsema, P. M.; Postma, T. J. B. M. (2009). Cooperative Innovation Projects: Capabilities and Governance Mechanisms. Journal of Product Innovation Management, 26 (1) $58-70$.

Carvalho, F. L., Diaz, M. D. M., Neto, S. B., \& Kalatzis, A. E. G. (2015). Saída e insucesso das cooperativas de crédito no Brasil: uma análise do risco. Revista Contabilidade \& Finanças, 26(67), 70-84.

Cheney, G., Santa Cruz, I., Peredo, A. M., \& Nazareno, E. (2014). Worker cooperatives as an organizational alternative: Challenges, achievements and promise in business governance and ownership. Organization, 21(5), 591-603.

Chueke, G. V., \& Amatucci, M. (2015). O que é bibliometria? Uma introdução ao fórum. Internext - Revista Eletrônica de Negócios Internacionais, 10(2), 1-5. 
Duarte, C. A. da S.; Jesus-Lopes, J. C. de \& Santos, L. M. R. dos. (2016, abril). Cooperativas: Um levantamento bibliométrico da produção científica do EnANPAD entre 2010-2014. Desafio Online, 4 (1).

Ferenhof, H. A.; Fernandes, R. F. (2014). Passos para construção da Revisão Sistemática e Bibliometria. v. 3.02. Recuperado em 14 de abril, 2019, de http://www.igci.com.br/artigos/passos_rsb.pdf

Freitas, A. F. de, \& Freitas, A. F. de (2014). O cooperativismo de crédito no Brasil e a emergência de uma vertente solidária. Revista Brasileira de Gestão e Desenvolvimento Regional, 10(2), 46-74.

Hagendorff, J. (2014). Corporate Governance in Banking. In A. N. Berger, P. Molyneux \& J. O. S. Wilson. The Oxford Handbook of Banking. Nova Iorque: Oxford University Press. Instituto Brasileiro de Governança Corporativa [IBGC]. (2015). Guia das Melhores Práticas de Governança para Cooperativas. São Paulo: Autor. Recuperado em 13 de abril, 2019, de

https://conhecimento.ibgc.org.br/Lists/Publicacoes/Attachments/22108/GuiaCoop_Web.p df.

International Co-operative Alliance [Coop]. (2019). Cooperative Principles. Recuperado em 13 de abril, 2019, de https://www.ica.coop/en/cooperatives/cooperativeidentity\#cooperative-values

Jansen, A., Maehler, A., \& Wegner, D. (2018). Cooperative Governance and the Legitimacy Dilemma: A Case Study in a Credit Cooperative. Iberoamerican Journal Of Strategic Management (IJSM), 17(3), 61-80.

Jensen, M. C.; Meckling, W. H. (1976). Theory of the firm: Managerial behavior, agency costs and ownership structure. Journal of Financial Economics, 3, 305-360.

Martins, P.; Lima, E. P.; Schenatto, F. J. A. \& Bortoluzzi, S. C. (2017, dezembro). Análise das características das publicações sobre avaliação de desempenho organizacional em cooperativas de crédito. Anais do Congresso Brasileiro de Engenharia de Produção, Ponta Grossa, PR, Brasil, 7.

Organização das Cooperativas Brasileiras [OCB]. (2019). Manual de Boas Práticas de Governança Cooperativa. Recuperado em 14 de abril, 2019, de https://www.ocb.org.br/publicacao/16/manual-de-governanca-cooperativa.

Peng, X., Liang, Q., Deng, W., \& Hendrikse, G. (2019). CEOs versus members' evaluation of cooperative performance: Evidence from China. The Social Science Journal. 56 (2). 55 63.

Revista Gestão e Secretariado (GeSec), São Paulo, SP, v. 11, n. 1, jan. / abr., 2020, p. 106-125. 
Pivoto, D., Waquil, P. D., Souza, M. de, \& Spanhol, C. P. (2015). Governança Cooperativa: O Problema do Horizonte em Cooperativas Agropecuárias do Rio Grande do Sul. Revista Brasileira de Gestão e Desenvolvimento Regional, 11(3), 206-228.

Quevedo-Silva, F., Santos, E. B. A., Brandão, M. M., \& Vils, L. (2016). Estudo bibliométrico: orientações sobre sua aplicação. Revista Brasileira de Marketing, 15(2), 246-262.

Seitz, F. (2012). The Cooperative Banking System in Germany: Empirical Evidence and Some Theory. Zeszyty Naukowe Wyższej Szkoły Bankowej w Poznaniu, (45 Dylematy rozwoju polskiej spółdzielczości kredytowej; Dilemmas of the Development of Cooperative Banking Credits in Poland), 77-86.

Shleifer, A., \& Vishny, R. W. (1997). A survey of corporate governance. The Journal of Finance, 52(2), 737-783.

Silva, J., Oliveira, C., Garcia, A., Sugano, J., \& Lucchesi, K. (2018). Um estudo bibliométrico de 1960 a 2017. Revista Vianna Sapiens, 9(1), 115-136.

Schulze, E. (1987). Estrutura do poder em cooperativas. Perspectiva Econômica, 22 (59), 4976.

Tomaz, D. A.; Serafin Junior, V.; Besen, F. G.; Almeida, R. S. de. (2018). Estudo de Publicações sobre a Avaliação de Desempenho e Eficiência das Cooperativas de Crédito e Bancos Públicos e Privados: Características Bibliométricas. Anais do Congresso brasileiro em Gestão de Negócios, 5. Unioeste, Cascavel, PR, Brasil, 2018.

Trindade, L. Z., \& Bialoskorski Neto, S. (2014). Análise e percepção dos custos das práticas de governança corporativa: um estudo de caso. Contextus - Revista Contemporânea de Economia e Gestão, 12(3), 64-97.

World Council of Credit Unions. (2019). Our Global Reach. 2019b. Recuperado em 13 de abril, 2019, de https://www.woccu.org/impact/global_reach.

Zylbersztajn, D. (1994). Organização de cooperativas: desafios e tendências. Revista de Administração, 29 (3), 23-32.

Submetido em: 14.04.2019

Aceito em: $\quad$ 15.08.2019 